\title{
Nasal Bone Fractures and the Use of Radiographic Imaging: An Otolaryngologist Perspective
}

Running Title: Nasal bone fractures and Radiology

Edward Westfall, MD ${ }^{1,2}$

Benton Nelson, $\mathrm{MD}^{1,2}$

Dominic Vernon, $M D^{1,2}$

Mohamad Z. Saltagi, MD ${ }^{1,2}$

Avinash V. Mantravadi, MD ${ }^{1,2}$

Cecelia Schmalbach, MD ${ }^{1,2}$

Jonathan $\mathrm{Y}$. Ting, MD, MS, MBA ${ }^{1,2}$

Taha Z. Shipchandler, MD ${ }^{1,2}$

Author Affiliations:

${ }^{1}$ Department of Otolaryngology—Head and Neck Surgery

${ }^{2}$ Indiana University School of Medicine

\section{Corresponding Author:}

Taha Z. Shipchandler, MD, FACS

Division Director - Facial Plastic, Aesthetic, \& Reconstructive Surgery

Associate Professor

Vice-Chair and Residency Director

Department of Otolaryngology_-Head and Neck Surgery, Indiana University Health Physicians, Indiana University School of Medicine 1130 W. Michigan Street, Suite 400, Indianapolis, IN 46202

Telephone: 317-278-1258

Fax: 317-274-8285

tshipcha@iupui.edu

Financial disclosures: none

Authors disclose no conflict of interest 


\section{Abstract}

Objective: To determine radiologic preferences of practicing otolaryngologists regarding isolated nasal bone fractures.

Study Design: An 8-question survey on isolated nasal bone fractures was designed.

Setting: Surveys were sent to all otolaryngology residency program directors for distribution among residents and faculty. Additional surveys were distributed to private practice otolaryngology groups. Subjects and Methods: Practicing academic (residents \& faculty) and private-practice otolaryngologists.

Results: 140 physicians responded to the survey. $57 \%$ of the respondents were practicing otolaryngologists ( $75 \%$ with $10+$ years of experience), while $43 \%$ of respondents were residents-intraining. $56 \%$ of respondents treated $1-5$ nasal bone fractures per month. $80 \%$ of all respondents reported imaging being performed prior to consultation. If imaging was obtained before consultation, plain films and computed tomography (CT) maxillofacial/sinus scans were the most frequent modalities. $33 \%$ of residents and $70 \%$ of practicing otolaryngologists report imaging as 'rarely' or 'never' helpful in guiding management. $42 \%$ of residents and $20 \%$ of practicing otolaryngologists report asking for imaging when it wasn't already obtained. Decreased use of radiography was associated with greater years in practice and higher frequency of fractures treated.

Conclusions and Relevance: Otolaryngologists seldom request imaging to evaluate and treat isolated nasal bone fractures. When ordered, imaging is utilized more often among residents-intraining and non-otolaryngology consulting physicians. This study highlights an opportunity to educate primary care and emergency room providers as well as otolaryngology residents on the value of comprehensive physical exam over radiographic imaging in the work-up of isolated nasal fractures. In addition, widespread adoption of a "no x-ray policy" in this setting may result in better resource utilization.

Level of Evidence: 3

Meeting Information: This work has not been presented previously.

Key Words: isolated, nasal bone fractures, trauma, radiography, CT maxillofacial, survey 


\section{Introduction}

Nasal bone fractures are exceedingly common in facial trauma due to the central location, prominent projection, and thin nature of the nasal bones ${ }^{1,2}$. These fractures most frequently occur in males in their $2^{\text {nd }}$ and $3^{\text {rd }}$ decades of life, but they also constitute $30 \%$ of pediatric facial fractures ${ }^{1-6}$. Left untreated, these fractures can lead to nasal obstruction including nasal valve collapse, cosmetic deformity and additional long term sequelae including chronic sinusitis and nasal growth retardation in children ${ }^{3,7}$. Concurrent septal injury can lead to further destabilization of the nose, nasal airway obstruction, lateral nasal tip deviation, and decreased tip projection in patients who are not properly treated with appropriate fracture reduction ${ }^{2,5}$.

The management of a nasal bone fracture is dependent upon the clinical presentation. Radiographic imaging is recommended in the setting of high energy craniomaxillofacial trauma to include loss of consciousness ${ }^{8}$. However, in the setting of isolated nasal trauma without evidence of other craniofacial injuries or trauma, plain film radiographs have repeatedly been shown to be noncontributory in further management ${ }^{9-}$ ${ }^{14}$. In settings where imaging is indicated due to other suspected injuries, the majority of the otolaryngologic literature advocates for a computed tomography (CT) maxillofacial scan as opposed to a facial xray ${ }^{5,6,9-15}$. Overall, CT scans may provide high resolution imaging of isolated nasal fractures, but it is debatable whether any imaging beyond the clinical exam improves patient management.

This study aims to evaluate the current radiographic practice patterns among otolaryngologists and residents who treat patients with isolated nasal bone fractures. We hypothesized that otolaryngologists in current practice rely upon physical examination as opposed to imaging in the identification and management of isolated 
nasal bone fractures. In addition, we hypothesized that consultants do not routinely obtain imaging for isolated nasal bone fractures, but primary care teams including emergency department (ED) providers routinely order such imaging as part of their work-up for isolated nasal trauma.

This study's importance lies in the fact that isolated nasal bone fractures are relatively common, and reliance on excessive imaging can amplify the cost of care ${ }^{14,16-}$ 18. Determining the use of radiography by otolaryngologists in such instances is vital for effective and cost-efficient management. 


\section{Methods}

The Indiana University Institutional Review Board approved this project. An anonymous, electronic survey was designed using the Google documents online survey creation tool (Google, Mountain View, CA). A copy of the final survey is shown in Figure 1. The survey identified respondent's years in practice, resident versus board certified otolaryngologist, the number of fractures regularly encountered, and the presence of imaging prior to consultation. Questions were incorporated to determine the role imaging plays in the consultant's management plan (e.g. frequency, modality, and reasons for request). Two hundred surveys were distributed to several otolaryngology training program directors in the United States as well as associated faculty and residents at their respective institutions. In addition, the study was distributed regionally to private otolaryngology practices. Incomplete surveys were not included in data analysis. Monetary and gift compensation was not provided for participation.

The survey data and demographics were analyzed using descriptive statistics. The primary outcome measure was the frequency, type, and utility of radiographic imaging in the work-up of isolated nasal fractures. Subgroup analysis was performed to identify potential practice pattern differences between otolaryngology residents in training versus practicing otolaryngologists. A two-tailed Chi-Squared analysis was used to determine resident and staff perceived differences in the utility of nasal fracture imaging. $P<0.05$ was deemed statistically significant. 
Results:

A total of 200 surveys were distributed. 140 surveys were completed, yielding a response rate of $70 \%$. Otolaryngology practice experience is summarized in Figure 2. $78(56 \%)$ respondents were in academic or private practice while the remaining 62 (44\%) were otolaryngology residents. The majority of respondents $(60 ; 43 \%)$ had 10 or more years of trauma experience post residency/fellowship training.

Most respondents (56\%) treated between 1-5 nasal bone fractures per month. In total, $80 \%$ reported imaging being 'always/often' obtained prior to otolaryngology consultation (Figure 3). Specifically, 39\% reported receiving plain films, while $61 \%$ reported prior CT imaging. In the event that imaging was desired, 110 respondents (79\%) requested maxillofacial/sinus CT (Figure 4). 42 (68\%) residents reported that imaging was helpful in their management of nasal fractures which was statistically higher than the 22 (28\%) practicing otolaryngologists who found imaging helpful $(p<0.001)$. Twenty-five $(42 \%)$ of residents and $16(20 \%)$ of practicing otolaryngologists reported asking for imaging when it was not already obtained. In addition, 33 (24\%) respondents reported 'never' obtaining imaging when consulted, while $62(44 \%)$ 'rarely' obtain imaging (Figure 5). 


\section{Discussion:}

This study demonstrates that otolaryngologists do not routinely order radiographic imaging to assess isolated nasal trauma. This supports previous findings by Logan et al who succinctly demonstrated that radiography rarely affects the treatment plan for those with an isolated nasal fracture ${ }^{17}$. The strength of this study lies in capturing current practices among otolaryngology residents, faculty, and nonotolaryngology physicians. We have importantly identified a high rate of radiographic imaging for isolated nasal trauma among consulting (non-otolaryngology) physicians, with nearly $40 \%$ of survey respondents stating that imaging was obtained by the consulting team prior to the involvement of the otolaryngologist. This finding identifies a knowledge gap and an opportunity for quality improvement.

It has previously been postulated that junior level residents less familiar with ideal practice standards contribute to the unnecessary use of plain films for nasal trauma work up ${ }^{14}$. An interesting finding in this study is the significant practice difference that exists between residents and non-residents within our own field of otolaryngology. Otolaryngology residents are more likely to order radiographs for isolated nasal bone injuries (42\%) compared to non-residents (20\%). Otolaryngology residents reported imaging to be useful in treatment planning whereas non-residents did not. Lastly, a trend towards increased use of CT imaging for the work up of nasal fractures was identified among residents, whereas non-residents were largely impartial to x-rays vs CT when examining nasal bone fractures. These data suggest that, as experience in clinical exam related to nasal bone injuries increases, the usefulness of radiographic 
imaging decreases. This highlights the importance of emphasizing clinical examination in the work up of isolated nasal fractures early in residency training.

In the case of complex facial trauma, CT findings often affect management strategies $^{12}$. However, current literature repeatedly emphasizes that CT imaging is not indicated for isolated nasal bone fractures unless concomitant symptoms or physical exam findings warrant a more robust work up ${ }^{1,16,18}$. Despite these recommendations, this study suggests that in current practice, CT maxillofacial/sinus scans are still routinely obtained for isolated nasal trauma prior to otolaryngology consultation. While $\mathrm{CT}$ is the preferred modality to assess complex facial trauma, consultants reported limited utility of CT imaging for isolated nasal fractures, and otolaryngologists overwhelmingly declined further imaging to evaluate a nasal bone fracture. Of the respondents who 'never' obtained imaging, the majority were board certified otolaryngologists with over 5 years of experience. In addition, as the number of nasal fractures seen per month increased, the utility of radiography decreased in our survey. This finding again suggests that clinical experience favors physical examination as opposed to imaging when diagnosing fractures.

The use of plain films in isolated nasal bone fractures is another important radiographic practice that has been studied in the past. Craniomaxillofacial trauma studies have emphasized that plain films have very limited use in the diagnosis and treatment of isolated nasal fractures ${ }^{10,12,14}$. Plain films have been shown to have high false positive rates for nasal fracture and septal deviation, and they can be difficult to interpret for radiologists and consultants alike $e^{1,8,15,19}$. In addition, nasal injuries are 
frequently associated with cartilaginous avulsions and separations which go undetected on plain films ${ }^{2}$.

Sharp et. al. conducted a prospective study analyzing the utility of plain film imaging in the management of isolated nasal trauma ${ }^{14}$. The authors failed to identify a case where the films altered treatment plan ${ }^{14}$. Their findings correlate strongly with the results of our survey. Their study led to a fundamental change in hospital policy with a creation of 'no x-ray policy' for isolated nasal trauma ${ }^{14}$. Given that the nasal bone is among the most, if the not the most, commonly fractured bones in the human body, this simple change in eliminating routing radiographic imaging, if applied on a wider scale, could lead to significant reduction in both cost and radiation exposure $1,7,8,16,18$.

The major limitations of this study are the selection and recall bias inherent to all survey studies. We attempted to be comprehensive in evaluating practice patterns by including otolaryngology residents, academic faculty, as well as private practitioners. In doing so, practice variations related to clinical setting were negated as much as possible. Another limitation of this study is that it does not answer the question of which patient group is more likely to undergo radiographic imaging. It would be especially useful to determine if children receive imaging more frequently base on difficultly performing a clinical exam or the higher risk of midfacial growth retardation ${ }^{4}$. It is possible that fear of potential litigation surrounding missed facial injuries contributes to overutilization of CT imaging, especially among children ${ }^{16}$. Our study does not help answer this question, and future studies would be helpful in investigating this further. 


\section{Conclusion}

Isolated nasal fractures are a common injury which can be diagnosed and treated effectively without radiographic imaging. Most otolaryngologists do not request imaging in order to identify and treat isolated nasal fractures. On the contrary, data from this survey demonstrates that imaging is frequently obtained prior to otolaryngology consultation (plain film radiographs and CT). Primary care services (first point of care) are more likely to obtain radiographic imaging compared to otolaryngologists. In addition, otolaryngology residents in training are more likely to rely on radiographic imaging compared to practicing otolaryngologists, especially those with multiple years of experience.

Ultimately, nasal bone plain films and CT scans have limited clinical utility in isolated nasal bone fracture management and unnecessarily add to healthcare costs. This study identifies a knowledge gap and an associated opportunity to educate primary care providers to include emergency room staff, as well as otolaryngology residents, in the work-up of isolated nasal fractures. This workup should emphasize a comprehensive physical exam over radiographic imaging. In addition, widespread national adoption of a "no x-ray policy" in the management of isolated nasal fractures will likely result in better resource utilization and improve the quality of patient care. 


\section{References}

1. Alvi A, Doherty $T$, Lewen G. Facial fractures and concomitant injuries in trauma patients. Laryngoscope. 2003;113(1):102-106.

2. Holt GR. Biomechanics of nasal septal trauma. Otolaryngol Clin North Am. 1999;32(4):615-619.

3. Carvalho TB, Cancian LR, Marques CG, Piatto VB, Maniglia JV, Molina FD. Six years of facial trauma care: an epidemiological analysis of 355 cases. Braz $\mathrm{J}$

Otorhinolaryngol. 2010;76(5):565-574.

4. Rohrich RJ, Adams WP, Jr. Nasal fracture management: minimizing secondary nasal deformities. Plast Reconstr Surg. 2000;106(2):266-273.

5. Anderson PJ. Fractures of the facial skeleton in children. Injury. 1995;26(1):47-50.

6. East CA, O'Donaghue G. Acute nasal trauma in children. J Pediatr Surg. 1987;22(4):308-310 .

7. Imahara SD, Hopper RA, Wang J, Rivara FP, Klein MB. Patterns and outcomes of pediatric facial fractures in the United States: a survey of the National Trauma Data Bank. J Am Coll Surg. 2008;207(5):710-716.

8. Finkle DR, Ringler SL, Luttenton CR, Beernink JH, Peterson NT, Dean RE. Comparison of the diagnostic methods used in maxillofacial trauma. Plast Reconstr Surg. 1985;75(1):32-41.

9. Chou C, Chen CW, Wu YC, Chen KK, Lee SS. Refinement treatment of nasal bone fracture: A 6-year study of 329 patients. Asian J Surg. 2015;38(4):191-198.

10. Clayton MI, Lesser TH. The role of radiography in the management of nasal fractures. J Laryngol Otol. 1986;100(7):797-801. 
11. Mondin V, Rinaldo A, Ferlito A. Management of nasal bone fractures. Am J Otolaryngol. 2005;26(3):181-185.

12. Nigam A, Goni A, Benjamin A, Dasgupta AR. The value of radiographs in the management of the fractured nose. Arch Emerg Med. 1993;10(4):293-297.

13. Rhee SC, Kim YK, Cha JH, Kang SR, Park HS. Septal fracture in simple nasal bone fracture. Plast Reconstr Surg. 2004;113(1):45-52.

14. Sharp JF, Denholm S. Routine X-rays in nasal trauma: the influence of audit on clinical practice. J R Soc Med. 1994;87(3):153-154.

15. de Lacey GJ, Wignall BK, Hussain S, Reidy JR. The radiology of nasal injuries: problems of interpretation and clinical relevance. Br J Radiol. 1977;50(594):412-414. 16. Illum P. Legal aspects in nasal fractures. Rhinology. 1991;29(4):263-266. 17. Logan M, O'Driscoll K, Masterson J. The utility of nasal bone radiographs in nasal trauma. Clin Radiol. 1994;49(3):192-194.

18. Turner BG, Rhea JT, Thrall JH, Small AB, Novelline RA. Trends in the use of CT and radiography in the evaluation of facial trauma, 1992-2002: implications for current costs. AJR Am J Roentgenol. 2004;183(3):751-754.

19. Adeyemo WL, Akadiri OA. A systematic review of the diagnostic role of ultrasonography in maxillofacial fractures. Int J Oral Maxillofac Surg. 2011;40(7):655661. 


\section{Figure Legends}

Figure 1. This is the complete 8-question survey that was distributing to otolaryngology residents, attendings at academic programs, and private-practice otolaryngologists.

Figure 2. This survey question asked practitioners about their years of experience treating nasal bone fractures. As demonstrated, $43 \%$ of survey respondents had more than 10 years of experience, and nearly $50 \%$ had at least 5 years of experience postresidency.

Figure 3. This survey question asked otolaryngologists how often imaging was obtained prior to consultation on a patient with an isolated nasal bone fracture. As demonstrated, $80 \%$ of otolaryngologists stated that imaging was "always" or "often" obtained prior to consultation. The imaging modalities obtained were plain films or maxillofacial computed tomography scans, or both.

Figure 4. This survey question asked survey respondents which imaging modality they would request if they were interested in obtaining imaging for nasal bone fracture. Nearly $80 \%$ of respondents stated that they preferred CT (computed tomography) Maxillofacial scans, while there was little interest in obtaining plain films.

Figure 5. This survey question asked how often otolaryngologists requested imaging. $68 \%$ of respondents stated that they 'never' or 'rarely' request imaging. 
1. How long have you been practicing otolaryngology?
a. More than 10 years post residency/fellowship training
b. $5-10$ years post residency/fellowship training
c. 1-5 years post-residence/fellowhip training
d. In residency/fellowship PGY-5
e. In residency/fellowship PGY-4
f. In residency/fellowship PGY-3
g. In residency/fellowship PGY-2
h. Other

2. How often do you encounter nasal fractures in your practice?
a. More than 10 cases per month
b. $5-10$ cases per month
c. $1-5$ cases per month
d. 1 case or less
e. never

3. How often is imaging already performed prior to consultation with your service?
a. Always
b. Often
c. Sometimes
d. Rarely
e. Never

4. If imaging is performed prior to consultation, what type of study is most commonly completed?
a. Plain films
b. CT maxillofacial/sinus
c. MRI
d. Other (please elaborate) 
5. If imaging was ordered prior to consultation, how often does this direct treatment planning?
a. Always
b. Often
c. Sometimes
d. Rarely
e. Never

6. How often do you ask for imaging?
a. Always
b. Often
c. Sometimes
d. Rarely
e. Never

7. Why do you request imaging?
a. Operative decision making
b. Classify fractures
c. Medico-legal implications
d. Other (please elaborate)

8. What imaging study if desired do you ask for?
a. Plain films
b. $\mathrm{CT}$ face/sinus
c. MRI
d. Other(please elaborate) 
How long have you been practicing otolaryngology?

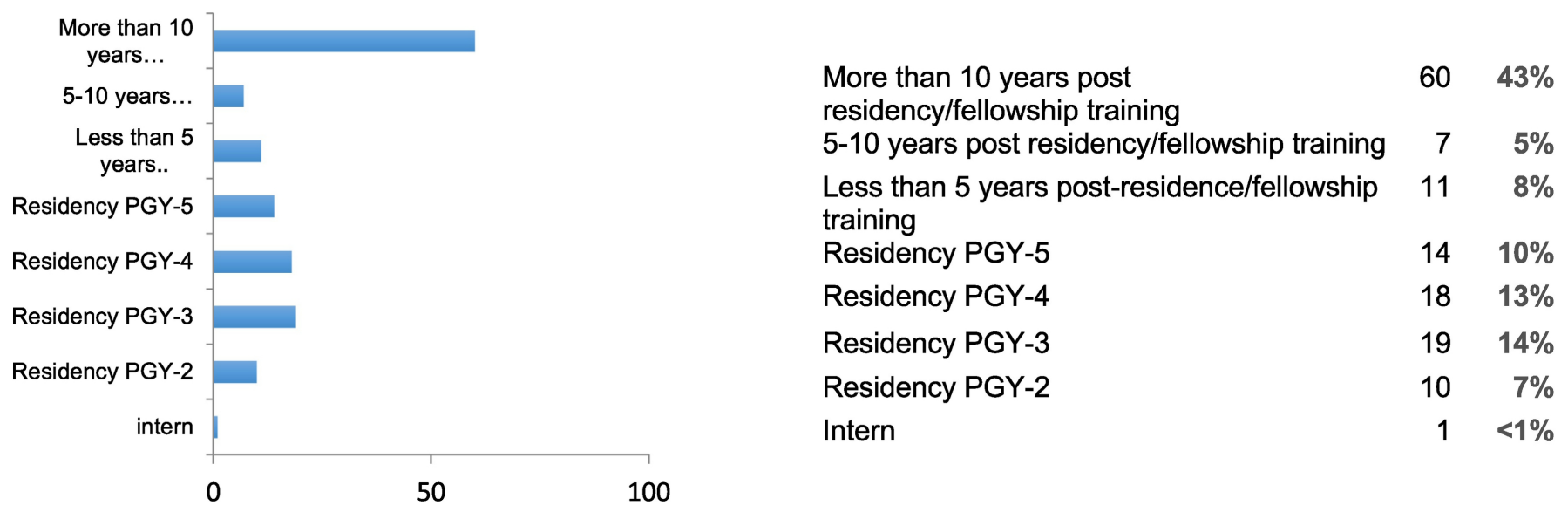


How often is imaging already performed prior to consultation with your service?

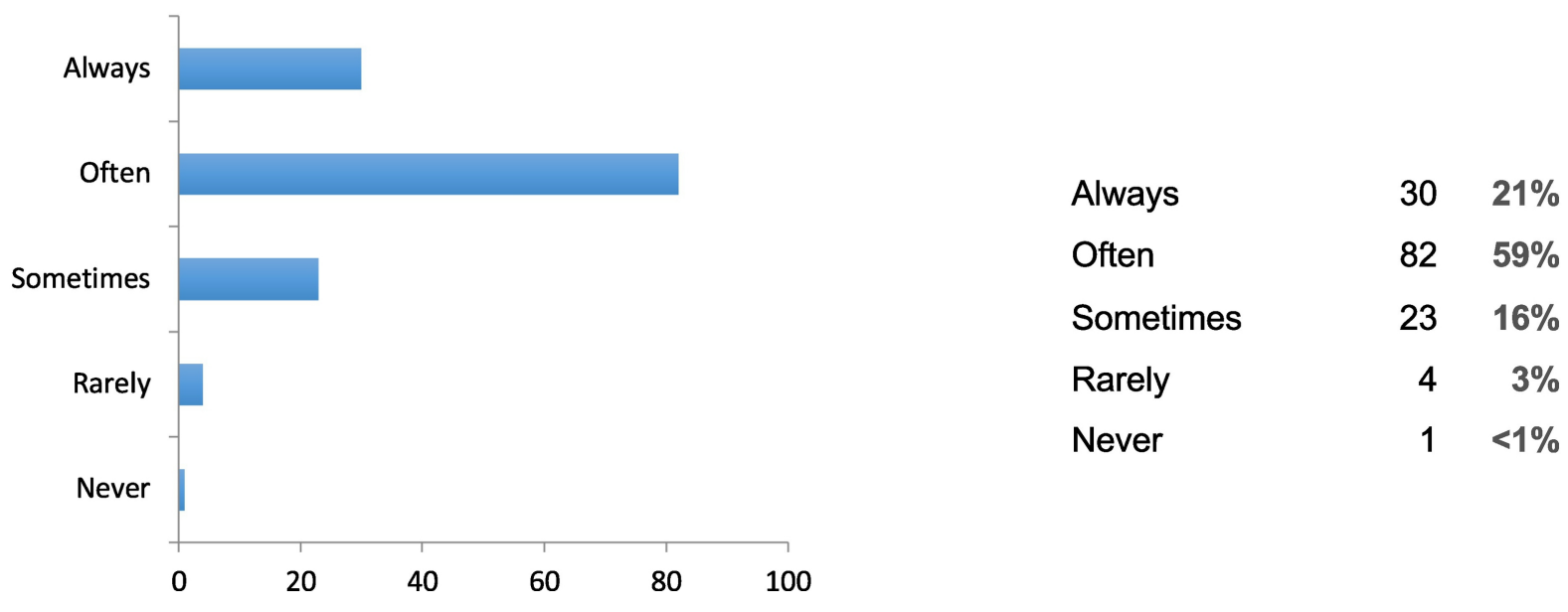


What imaging study, if desired, do you ask for?

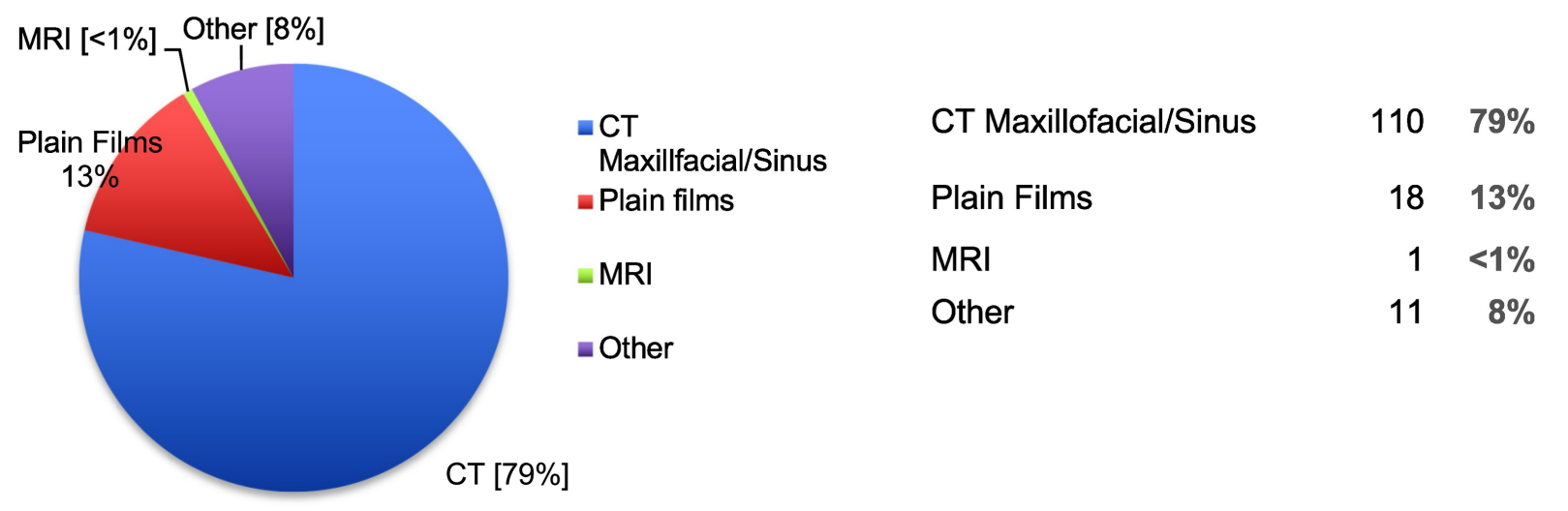


How often do you ask for imaging?

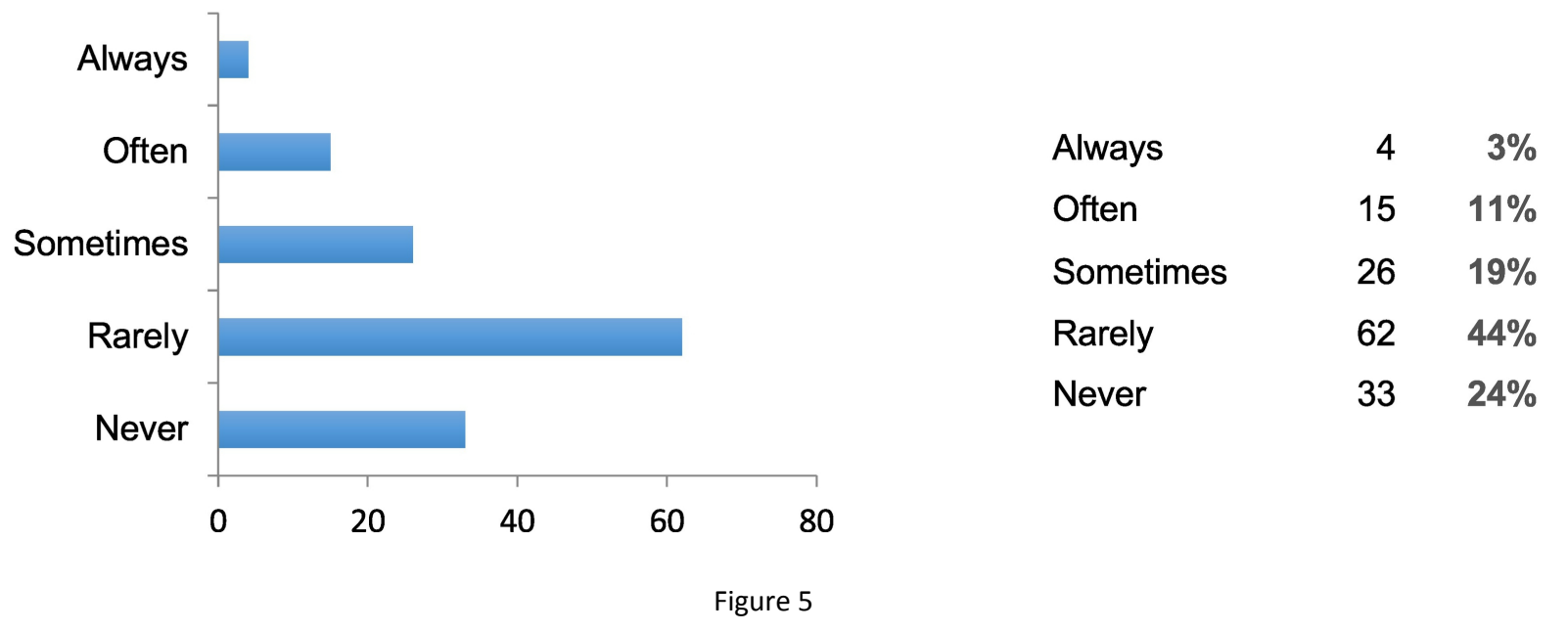

